\title{
BMJ Open Research priorities for the management of broken bones of the upper limb in people over 50: a UK priority setting partnership with the James Lind Alliance
}

Warren J Sheehan (D) , ${ }^{1}$ Mark A Williams (D) , ${ }^{2}$ Zoe Paskins, ${ }^{3,4}$ Matthew L Costa, ${ }^{5}$ Miguel Antonio Fernandez (1) , ${ }^{1,6}$ Jenny Gould, ${ }^{7}$ Philip Bell, ${ }^{8}$ Liz Baird, ${ }^{9}$ Richard Grant, ${ }^{10}$ Patricia Ellis, ${ }^{11}$ Catherine White, ${ }^{12}$ Laura Arnel, ${ }^{1}$ Lauren Exell, Stephen Gwilym ${ }^{1}$

To cite: Sheehan WJ, Williams MA, Paskins Z, et al. Research priorities for the management of broken bones of the upper limb in people over 50: a UK priority setting partnership with the James Lind Alliance. BMJ Open 2019;9:e030028. doi:10.1136/ bmjopen-2019-030028

- Prepublication history and additional material for this paper are available online. To view these files, please visit the journal online (http://dx.doi org/10.1136/bmjopen-2019030028).

Received 27 February 2019 Revised 06 June 2019 Accepted 26 July 2019

Check for updates

(c) Author(s) (or their employer(s)) 2019. Re-use permitted under CC BY-NC. No commercial re-use. See rights and permissions. Published by BMJ.

For numbered affiliations see end of article.

Correspondence to

Warren J Sheehan;

warren.sheehan@ouh.nhs.uk

\section{ABSTRACT}

Objective To determine research priorities for the management of broken bones of the upper limb in people over 50 , which represent the shared priorities of patients, their families, carers and healthcare professionals.

Design/setting A national (UK) research priority setting partnership.

Participants People aged 50 and over who have experienced a fracture of the upper limb, carers involved in their care, family and friends of patients, healthcare professionals involved in the treatment of these patients. Methods Using a multiphase methodology in partnership with the James Lind Alliance over 15 months (September 2017 to December 2018), a national scoping survey asked respondents to submit their research uncertainties. These were amalgamated into a smaller number of research questions. The existing evidence was searched to ensure that the questions had not already been answered. A second national survey asked respondents to prioritise the research questions. A final shortlist of 25 questions was taken to a multi-stakeholder workshop where a consensus was reached on the top 10 priorities.

Results There were 1898 original uncertainties submitted by 328 respondents to the first survey. These original uncertainties were refined into 51 research questions of which 50 were judged to be true uncertainties following a review of the research evidence. There were 209 respondents to the second (interim prioritisation) survey. The top 10 priorities encompass a broad range of uncertainties in management and rehabilitation of upper limb fractures.

Conclusions The top 10 UK research priorities highlight uncertainties in how we assess outcomes, provide information, achieve pain control, rationalise surgical intervention, optimise rehabilitation and provide psychological support. The breadth of these research areas highlights the value of this methodology. This work should help to steer research in this area for the next 5-10 years and the challenge for researchers now is to refine and deliver answers to these research priorities.

\section{Strengths and limitations of this study}

- This is the first time healthcare professionals have worked alongside patients and carers to set priorities for upper limb fracture research using the established James Lind Alliance methodology.

- Survey responses were received from all over the UK, with an even split of healthcare professionals and members of the public (patients, carers, family and friends).

- Although substantial effort went into recruitment, ethnic minority groups were still under-represented.

- While the research priorities are now reported, it is up to the research community and research funding organisations to refine and deliver the answers to these questions.

\section{INTRODUCTION}

Upper limb fractures are very common injuries ${ }^{1}$ with distal radius and proximal humerus fractures being the most prevalent. ${ }^{23}$ Of these injuries, over $60 \%$ of all distal radius and $75 \%$ of all proximal humerus fractures occur in people over $50 .^{3}$ These fractures can arise from both high energy trauma, such as a road traffic accident, and low energy trauma, such as falling from a standing height-sometimes called fragility fractures. As the population ages, the incidence of these types of upper limb fractures is increasing. ${ }^{4}$

Fractures of the upper limb have significant cost implications to the health and social care services. ${ }^{15}$ They often require both outpatient and inpatient care, and treatment may involve surgery. They also have financial and social implications for the people affected and their families. ${ }^{15}$ Many people over 50 do not return to their pre-injury level of function 
following a fracture and often report long-term impaired function, fear of falling and associated disability. ${ }^{15}$

Research is integral in shaping the future of healthcare provision. However, the research being undertaken does not always match the research priorities of patient $\mathrm{s}$ and healthcare professionals. ${ }^{9-11}$ This can lead to a waste of time, money and other resources. ${ }^{12}$ There has been a strong drive to rectify this issue in recent years, and there is good evidence that patient and public involvement (PPI) in research has enhanced the quality and appropriateness of research. ${ }^{13-15}$ It has also helped to ensure that study findings are disseminated in a user-friendly way. ${ }^{13-15}$ PPI is now a key part of the design, conduct and delivery of research in health and social care. ${ }^{1617}$

The James Lind Alliance (JLA) is a non-profit organisation hosted by the National Institute for Health Research (NIHR). It provides a valuable means by which both patients and clinicians can shape the health research agenda. The aim of the JLA is to enable patients, carers and healthcare professionals to work together on an equal footing and to identify, prioritise and agree which research uncertainties are most important to them. ${ }^{18} 19$ To date, there have been over 50 priority setting partnerships (PSPs) across a range of disciplines, with over 100 research topics addressed as a direct result of the JLA PSPs. ${ }^{19-21}$

Although there are existing research recommendations in guidelines from the National Institute of Health and Care Excellence (NICE) and the British Society of Surgery for the Hand (BSSH), ${ }^{22} 23$ there has not, until now, been a national priority setting exercise for the management of broken bones of the upper limb in people over 50 . The aim of this project was to use the JLA process to obtain the views of patients, carers and healthcare professionals in order to establish, and then disseminate, their agreed top 10 research priorities. These priorities will drive future research into the best management of broken bones of the upper limb in people over 50.

\section{METHODS}

The 'Broken Bones of the Upper Limb in People over 50' $\mathrm{PSP}$ was conducted in accordance with the JLA process ${ }^{18} 19$ and was undertaken over a 15-month period (September 2017 to December 2018). See figure 1 'Time Frame of Priority Setting Partnership'.

\section{Steering group and partner organisations}

Steering group members were recruited to ensure that there was a good representation of PPI members and healthcare professionals including medics and allied health professionals. Each member had an interest in upper limb fractures and links to relevant partner organisations (see supplementary appendix 1-partner organisations). This ensured that a range of stakeholder groups was represented.

The steering group was supported by a JLA adviser with local administrative support. The JLA adviser was assigned as an independent neutral facilitator to ensure that each member had an equal opportunity to contribute to discussions and decisions and to ensure that the PSP followed the JLA process in a fair and transparent way. The information specialist designed the surveys, managed the data and performed the analysis, with each task being overseen and advised on by the steering group.

\section{Definition of scope}

The scope of this PSP covered any uncertainty relating to upper limb fractures, including those of the scapula, clavicle, humerus, radius and ulna bones, in people over 50 . Although fractures of the hand and carpal bones would also fall under the definition of upper limb fractures, it was

\begin{tabular}{|ll|}
\hline Prior to September 2017 & $\begin{array}{l}\text { Recruitment of healthcare professionals and members of the public to the PSP } \\
\text { steering group. } \\
\text { September } 2017\end{array}$ \\
Initial face-to-face meeting to agree partner organisations, the terms of \\
reference (TOR) and to determine the protocol and scope of the PSP. \\
Monthly teleconferences to sign off TOR, protocol and scope and to \\
design initial survey.
\end{tabular}

Figure 1 Time frame of priority setting partnership (PSP). 
agreed that they would not be a part of this PSP as they had already been covered by the JLA PSP on 'Common Conditions Affecting the Hand and Wrist'. ${ }^{24}$ We excluded uncertainties about chronic regional pain syndrome, too, as they were also included in the hand and wrist PSP. ${ }^{24}$

Prevention of primary and secondary fractures (including uncertainties about osteoporosis) was excluded from this PSP as it was felt that this is a very extensive and important area that should have its own priority setting project. Management and prevention of falls were excluded, though uncertainties involving fear of falling following an upper limb fracture were included.

Research recommendations from other sources, such as NICE and BSSH guidelines, ${ }^{22}{ }^{23}$ were not automatically included but were considered in establishing areas of uncertainty.

\section{Initial survey design and dissemination}

An initial survey asked patients, carers and healthcare professionals what they thought were important questions relating to upper limb fractures in people over 50 . The survey was designed as both a paper version and an online version via Online Surveys (formerly Bristol Online Surveys).$^{25}$ The survey enabled people to use free text and was set out in three phases of management (early treatment, treatment of the fracture, recovery and rehabilitation) with examples, to help people think of areas in which they may wish to include uncertainties. A fourth free text section was included to enable respondents to add any uncertainties they thought did not fit into the previous sections. The design and wording were developed by the PPI members to ensure it was user-friendly for the general population and that it did not influence responses. Both versions were piloted to healthcare professionals, patients and families, and their feedback was used to produce the final version. Some basic demographic information such as gender, initial section of postcode and whether they would describe themselves as a patient, family member, carer or healthcare professional was also collected.

The initial survey was disseminated by each member, via both personal contacts and their own partner organisations. The latter included relevant healthcare charities, specialist societies and user groups (see supplementary appendix 1-partner organisations). The survey was advertised in general practitioner surgeries and outpatient clinics via posters and flyers, and paper versions were left for people to complete with freepost return envelopes. Some steering group members were able to advertise the survey in newsletters and on the radio.

\section{Collection of uncertainties and identification of common themes}

The responses to the initial survey were collected and recorded in their unedited form. Responses that were not submitted as a question were still included where possible and were reworded by the information specialist into an uncertainty, without changing the content of the response. These alterations were agreed by the steering group who had access to the unedited uncertainty.

Once the responses were collated, general comments and out-of-scope uncertainties were removed and uncertainties with similar meaning were grouped together under an initial question. These questions were then placed under overarching themes.

\section{Creation of indicative questions}

The steering group met to discuss the overarching themes and each of the initial questions. Similar questions were amalgamated into an 'indicative question' that was broader in scope than each of the original questions. For example, 'Is a shoulder hemiarthroplasty or reverse total shoulder replacement better for a proximal humeral fracture in people over 50?' and 'What is the optimal surgical management of distal clavicle fractures in people over 50?' were both included under the indicative question, 'What is the best surgical management for upper limb fractures in people over 50? (incision, technique, metalwork, technology)'. The PPI members of the group ensured that the questions were written in plain English and that they were understandable to the general public.

\section{Literature review}

Following the creation of the indicative questions, literature reviews were undertaken to ensure that they were 'true' uncertainties that had not already been answered by research.

As per JLA guidelines, we deemed a question to be unanswered if either (1) no recent (within the past 3 years) reliable systematic reviews of research evidence addressing the question existed or (2) up-to-date systematic reviews of research evidence showed that uncertainty still persists. ${ }^{18} 19$ The steering group agreed that if there were no up-to-date systematic reviews, other sources of evidence should be searched. These included larger randomised control trials, either published or in progress, and published national evidence-based guidelines. Results of these were discussed with the experts on the steering group and a consensus was agreed. The indicative question was deemed to be answered if the evidence was high quality, did not show any further uncertainty and specifically answered the question.

The information specialist searched the following databases/sources of information:

- OpenAthens (https://openathens.nice.org.uk)-databases included PubMed, CINAHL, AMED, BNI, EMBASE, HBE, Medline and PschINFO.

- The Cochrane Central Register of Controlled Trials (https://www.cochranelibrary.org)

- Google scholar (https://scholar.google.co.uk).

- The WHO International Clinical Trials Registry Platform Search Portal (http://www.who.int/ictrp/en).

- Current Controlled trials (http://www.isrctn.com)

- The US National Institute of Health Trials Registry (https://clinicaltrials.gov).

- Published UK national guidelines. ${ }^{22} 23$ 


\section{Interim prioritisation}

Following the evidence-checking phase, an interim prioritisation survey was created, which included the remaining indicative questions. Wording was agreed by the steering group to ensure that it used easy to understand language and was in a user-friendly format. A 5-point Likert scale was used to rate the importance of each question ranging from 'not important' through to 'extremely important. A point score was given to each answer to help with the prioritisation phase, and this was agreed by the steering committee. This survey was once again made available in both paper and online versions, the latter via Online Surveys. ${ }^{25}$ It was distributed via the same channels as the initial survey.

The steering group agreed that there should be 25 indicative questions taken forward to the final workshop. Once all responses were collated, they were scored according to a preagreed scoring system. The indicative questions were ranked in three tables: (1) using all of the responses; (2) using only the responses from healthcare professionals; and (3) using only the responses from the patients/family/carers.

It was agreed that any question that was ranked in the top 25 in all three tables would automatically be included. Any question that ranked lower than 30 in all three groups would automatically be discarded. Any remaining questions would be considered on an individual basis.

\section{Final workshop}

Healthcare professionals and members of the public came together for a 1-day workshop to ascertain the final 'Top 10' research questions that would be presented to research funders. Participants were recruited via an invitation circulated by the steering group members, utilising their partner organisations networks. People were allocated places on a first come, first served basis with a cap on each group (patients, carers, healthcare professionals) to ensure equal numbers.

The 25 questions were discussed and ranked, and any agreements and disagreements were noted. There were two rounds of discussions in smaller groups. Participants were allocated into different groups for each round, but the balance of healthcare professionals and members of the public was maintained. A final decision-making discussion with the whole group determined the final 'Top 10' research priorities and was agreed by consensus.

\section{Patient and public involvement}

PPI was essential in the successful completion of this project. Having an equal representation of patient and public (PP) and healthcare professional (HP) members on the steering group is key to the JLA methodology and our steering group consisted of four (4) PP representatives.

They helped to set the remit and scope of this PSP in the initial stages as well as overseeing the study and ensuring that all surveys were in an easy to understand language and format. Their participation in the final workshop ensured that the patient and carer voice was represented in the final prioritisation. They will also play an integral part in the dissemination process through both social media and their partner organisations.

Each PP member made particular efforts to approach a diverse range of patient and carer groups across a number of settings to encourage responses to the surveys.

\section{Dissemination}

The dissemination strategy of this work was discussed and agreed by the steering group, and the publication of this paper will form the main distribution method. It will be disseminated to funding and research agendasetting organisations, as well as to partner organisations and PPI groups. The results will be presented at trauma conferences, to other medical interest groups, as well as on social media forums. They will be placed in trauma clinic waiting rooms across the UK and uploaded on to the JLA website as a point of open access.

\section{Ethics statement}

This work did not require ethics approval as per the JLA guidance and guidance published by the NHS National Patient Safety Agency National Research Ethics Service.

\section{RESULTS}

\section{Initial survey}

Three hundred and twenty-eight respondents submitted 1898 comments and original uncertainties. Forty-two per cent $(\mathrm{n}=138)$ of respondents were healthcare professionals, $53 \%(\mathrm{n}=174)$ were members of the public and $5 \%$ $(\mathrm{n}=16)$ preferred not to say. There were responses from across the UK with the highest single number being from Oxfordshire $(n=75,23 \%)$. There was a female to male ratio of 2:1 $(\mathrm{n}=202: 103)$, which may reflect the higher incidence of upper limb fractures in women over $50 .{ }^{3}$ The majority of respondents $(\mathrm{n}=268)$ classified themselves as white $(82 \%)$ with $10 \%(\mathrm{n}=34)$ from minority ethnic groups and $8 \%$ preferring not to say $(\mathrm{n}=26)$.

After 60 general comments and 114 out-of-scope uncertainties were removed, 1724 original uncertainties remained. Duplicate and similar original uncertainties were grouped together to form 246 initial questions.

After reviewing each of the initial questions, similar questions were combined to formulate 51 'indicative' questions. Seven (7) overarching themes were agreed: imaging, information, management, pain, prognosis, psychology and rehabilitation. Following the literature search, only one of these indicative questions was thought to have enough evidence to rule it out as a 'true' uncertainty, leaving 50 indicative questions for the interim prioritisation survey.

\section{Interim prioritisation survey}

There were 209 responses to the interim survey. Fortynine per cent of respondents were healthcare professionals $(\mathrm{n}=102)$ and $51 \%$ were members of the public $(\mathrm{n}=107)$. There were responses from across the UK. The 
majority of respondents $(\mathrm{n}=187)$ classified themselves as white $(90 \%)$ with $8 \%$ from minority ethnic groups $(n=17)$ and $2 \%$ preferring not to say $(n=4)$.

The three ranking tables were reviewed by the steering group. Twenty questions ranked in both the healthcare professional and the members of the public groups' top 25 so were immediately chosen to be in the final 25 questions. Twenty questions ranked outside the top 30 in both groups, so were excluded from the final 25 questions.

The remaining 10 questions were discussed by the steering group, with the following considerations being borne in mind:

- Their ranking in both groups;

- Their ability to be answered in research.

- Whether they were already covered by a research recommendation in existing guidelines (with priority being given to questions not previously published).

- Whether they already featured in any ongoing trials.

The remaining five questions were chosen, and the 25 questions for the final workshop were agreed by consensus.

Supplementary appendix 2 (flowchart of priority setting partnership and number of questions at each stage) highlights the stages and processing of questions.

\section{Final workshop}

The final workshop was attended by seven healthcare professionals (including surgeons, a GP, nurses and allied health professionals) and 13 members of the public (including patients who had suffered an upper limb fracture, carers for patients who had sustained an upper limb fracture and PPI members of the steering group). It was facilitated by three JLA representatives.

During the third round of discussions, it was decided that two of the preliminary 'Top 10' questions were very similar and could be combined by changing the wording slightly, without changing the overall meaning of the questions, as follows:

1. What are the most important outcomes after an upper limb fracture in people over 50 ? (eg, time for the bone to heal/return to normal activities/time to achieve a good recovery/cosmetic appearance)

2. What physical, psychological and financial effects do upper limb fractures have on patients over 50 and their families?

Combined wording:

- What are the most important outcomes after an upper limb fracture in people over 50 including physical, psychological and financial effects? (eg, time for the bone to heal/return to normal activities/time to achieve a good recovery/cosmetic appearance)

The order of the final 10 priorities was debated, and the final results were agreed by a majority consensus. They are shown in figure 2: final top 10 research priorities.

The full list of the top 24 can be viewed in supplementary appendix 3: 'Top 24 Research Priorities following Final Workshop'.
The full list of indicative research questions, and the original uncertainties which formulated these questions, may be found at http://www.jla.nihr.ac.uk/prioritysetting-partnerships/broken-bones-of-the-upper-limb/ on the JLA website.

\section{DISCUSSION}

The JLA process has enabled this PSP to successfully identify the 'Top 10' research priorities for the management of broken bones of the upper limb for people over 50 . These questions represent a broad range of priorities covering important topics such as outcomes, information, pain control, surgical intervention, rehabilitation and the need for psychological support. This range of topics was achieved due to the strong interactions between healthcare professionals and members of the public, especially at the final workshop. This was evidenced by the unanimous consensus of combining two of the questions during the final stage of prioritisation, thus allowing another important priority to be included in the 'Top 10'.

This study had a number of strengths, including use of the established JLA methodology (both qualitative and quantitative) itself. This ensured that the process was fair and transparent and that the priorities represented the shared interests of healthcare professionals, carers, patients and their families. Although there have been previous prioritisation studies involving the upper limb, ${ }^{24}{ }^{26}$ and for osteoporotic fractures generally, ${ }^{27}$ this was the first study to report national research priorities in fractures of the upper limb for people over 50 . The even split in responses between healthcare professional and members of the public ensured that the priorities were a true representation of all stakeholders.

Some respondents felt that some of the indicative questions were too broad and could potentially involve some loss of detail of the initial questions and original uncertainties. Although the original uncertainties and initial questions were more specific, the 246 initial questions would have been too difficult to prioritise, and so, as per JLA guidelines, ${ }^{18}$ the broader indicative questions needed to be developed. This issue has also been raised in other PSPs,${ }^{2021}$ however, Fernandez et $a l^{21}$ found that the "more specific' questions often ranked lower than the broader, 'less specific' questions, and so using broader questions was in fact seen as a strength in this particular study.

The 25 questions that were discussed and prioritised at the final workshop encompassed 1386 of the 1724 original, in-scope uncertainties. The remaining uncertainties either being answered previously on literature review or deprioritised by the JLA process. We feel that this analysis to assess inclusivity justifies the decision to include the broader indicative questions. It shows that the prioritised questions are an appropriate representation of what stakeholders wanted to know about the management of broken bones of the upper limb in people over 50 .

To help prevent any loss of detail and to also help researchers and research funders, we have included each of 
1. What are the most important outcomes after an upper limb fracture in people over 50 including physical, psychological and financial effects? (e.g. time for the bone to heal / return to normal activities / time to achieve a good recovery / cosmetic appearance)

2. What type of information should patients over 50 with an upper limb fracture be given and how should this be provided? (e.g. nutrition, pain relief, rehabilitation, return to work, driving, sport / exercise)

3. What is the best physical rehabilitation programme for people over 50 with an upper limb fracture (with or without surgery) when it NO LONGER needs to be kept still?

4. In people over 50, what is the patient experience of an upper limb fracture management and how does it influence outcomes?

5. What is the best alternative to medication / drugs to manage pain following an upper limb fracture in people over 50 ? (e.g. splints / casts / lining the fracture back up / ice)?

6. What is the best physical rehabilitation programme for people over 50 with an upper limb fracture (with or without surgery) whilst it needs to be kept still (e.g. in a cast or sling)?

7. Which are the most important factors which decide whether a patient over 50 with an upper limb fracture is treated with or without surgery?

8. What is the best surgical management for an upper limb fracture in people over 50 ? (e.g. incision, technique, metalwork, technology)

9. What is the best way of treating people over 50 who are at risk of developing psychological issues following an upper limb fracture?

10. For people over 50 , how well does a wrist fracture need to be reduced (put back into its normal position) to ensure a good long-term functional outcome?

Figure 2 Final top 10 research priorities.

the initial, more specific, questions under each of the indicative questions, in addition to all the original uncertainties, including those that were deemed to be out of scope, on the JLA website. This ensures transparency and provides open access to anyone who is interested in upper limb fracture research. It also gives those interested the opportunity to address not only the 'Top 10' priorities but also any of the original uncertainties.

In this PSP, there was some difficulty in obtaining high numbers of responses from across all regions of the UK. Much effort was made to publicise the surveys as widely as possible, and although we received responses from many different counties, both surveys had a larger number of responses from the counties where steering group members were based. Although the number of responses for both surveys was not high in comparison to those of some PSPs, ${ }^{20}$ this project did compare well to many others. ${ }^{21}{ }^{26}$ Considerable effort was made by all steering group members to engage patients and healthcare professionals via their partner organisations, general practitioner surgeries, hospital outpatient clinics, and families and friends; however, there was significant difficulty in recruiting from ethnic minority groups. Therefore, it should be noted that priorities that were identified in this study may not be a true representation of the views of these groups.

The 'Top 10' research priorities for the management of broken bone of the upper limb in people over 50 that have been identified by this study will now be disseminated as widely as possible. This includes dissemination to funding and research agenda-setting organisations such as the NIHR Evaluation, Trials and Studies Coordinating Centre (NETSCC), which includes the NIHR Health Technology Assessment Programme, and the Medical Research Council, as well as the major research funding charities. Publication of this paper will serve as the main distribution method and will be sent to all stakeholders and PPI groups. The results will be presented at trauma conferences, to other medical interest groups, as well as on social media forums. They will be placed in trauma clinic waiting rooms across the UK and uploaded on to the JLA website as a point of open access.

We anticipate that the research priorities that have been identified by this project will help shape the research in this area for many years to come and that this will in turn lead to improved quality of care for all patients. 
Author affiliations

${ }^{1}$ Oxford Trauma, Nuffield Department of Orthopaedics, Rheumatology and

Musculoskeletal Sciences, University of Oxford, Oxford, UK

${ }^{2}$ Sport, Health Sciences and Social Work, Oxford Brookes University, Oxford, UK

${ }^{3}$ Primary Care Centre Versus Arthritis, Research Institute for Primary Care \& Health

Sciences, Keele University, Keele, UK

${ }^{4}$ Haywood Academic Rheumatology Centre, Midlands Partnership NHS Foundation Trust, Stoke-on-Trent, UK

${ }^{5}$ Nuffield Department of Orthopaedics, Rheumatology and Musculoskeletal

Sciences, University of Oxford, Oxford, UK

${ }^{6}$ Trauma and Orthopaedic Surgery, University Hospitals Coventry and Warwickshire NHS Trust, Coventry, UK

${ }^{7}$ Patient Representative, London, UK

${ }^{8}$ Patient Representative, Isle-of-Anglesey, Wales, UK

${ }^{9}$ Patient Representative, Oxford, UK

${ }^{10}$ Patient Representative, Southampton, UK

${ }^{11}$ James Lind Alliance, Southampton, UK

12James Lind Alliance (until March 2018), Southampton, United Kingdom

Acknowledgements The authors wish to acknowledge the patients, their families and friends, carers and healthcare professionals who submitted responses to the national surveys. In addition, they would like to thank the partner organisations (supplementary appendix 1) who supported and promoted this work, the JLA for support and guidance throughout the process and all attendees at the final workshop who worked tirelessly to achieve a consensus on the top 10 research questions.

Contributors All authors (WJS, MAW, ZP, MLC, MAF, JG, PB, LB, RG, PE, CW, LA, LE and $\mathrm{SG}$ ) made significant contributions to the design, implementation and delivery of this research. MAW, ZP, MLC, MAF, JG, PB, LB, RG, PE, LE and SG provided significant edits to the manuscript and approved the data analysis. All authors have read and approved the final version of this manuscript. WJS analysed the data and prepared the initial manuscript.

Funding The Partnership was funded by the Orthopaedic Trauma Society and the British Orthopaedic Association and was supported by the National Institute for Health Research (NIHR) Oxford Biomedical Research Centre (BRC).

Disclaimer The views expressed are those of the authors and not necessarily those of the National Health Service, the National Institute for Health Research or the Department of Health and Social Care.

Competing interests MLC is a member of the NIHR HTA General Board. ZP is funded by the NIHR (Clinician Scientist Award (CS-2018-18-ST2-010)/NIHR Academy).

Patient consent for publication Not required.

Ethics approval This work did not require ethics approval as per the JLA guidance and guidance published by the NHS National Patient Safety Agency National Research Ethics Service.

Provenance and peer review Not commissioned; externally peer reviewed.

Data availability statement Data are available in a public, open access repository.

Open access This is an open access article distributed in accordance with the Creative Commons Attribution Non Commercial (CC BY-NC 4.0) license, which permits others to distribute, remix, adapt, build upon this work non-commercially, and license their derivative works on different terms, provided the original work is properly cited, appropriate credit is given, any changes made indicated, and the use is non-commercial. See: http://creativecommons.org/licenses/by-nc/4.0/.

\section{ORCID iDs}

Warren J Sheehan http://orcid.org/0000-0002-4953-3110

Mark A Williams http://orcid.org/0000-0002-3488-847X

Miguel Antonio Fernandez http://orcid.org/0000-0003-1533-8752

\section{REFERENCES}

1 Sabesan VJ, Valikodath T, Childs A, et al. Economic and social impact of upper extremity fragility fractures in elderly patients. Aging Clin Exp Res 2015;27:539-46.
2 Pogue DJ, Viegas SF, Patterson RM, et al. Effects of distal radius fracture malunion on wrist joint mechanics. J Hand Surg Am 1990;15:721-7

3 Court-Brown CM, Caesar B. Epidemiology of adult fractures: a review. Injury 2006;37:691-7.

4 Friedman SM, Mendelson DA. Epidemiology of fragility fractures. Clin Geriatr Med 2014;30:175-81.

5 Iglesias CP, Manca A, Torgerson DJ. The health-related quality of life and cost implications of falls in elderly women. Osteoporosis International 2009;20:869-78.

6 Pietri M, Lucarini S. The orthopaedic treatment of fragility fractures. Clin Cases Miner Bone Metab 2007;4:108-16.

7 Svedbom A, Borgstöm F, Hernlund E, et al. Quality of life for up to 18 months after low-energy hip, vertebral, and distal forearm fracturesresults from the ICUROS. Osteoporos Int 2018;29:557-66.

8 Edwards BJ, Song J, Dunlop DD, et al. Functional decline after incident wrist fractures--Study of Osteoporotic Fractures: prospective cohort study. BMJ 2010;341:c3324.

9 Crowe S, Fenton M, Hall M, et al. Patients', clinicians' and the research communities' priorities for treatment research: there is an important mismatch. Res Involv Engagem 2015;1.

10 Tallon D, Chard J, Dieppe P. Relation between agendas of the research community and the research consumer. Lancet 2000;355:2037-40.

11 Boddy K, Cowan K, Gibson A, et al. Does funded research reflect the priorities of people living with type 1 diabetes? a secondary analysis of research questions. BMJ Open 2017;7:e016540.

12 Chalmers I, Bracken MB, Djulbegovic B, et al. How to increase value and reduce waste when research priorities are set. Lancet 2014;383:156-65.

13 Thompson J, Bissell P, Cooper CL, et al. Exploring the impact of patient and public involvement in a cancer research setting. Qual Health Res 2014;24:46-54.

14 Mockford C, Staniszewska S, Griffiths F, et al. The impact of patient and public involvement on UK NHS health care: a systematic review. Int J Qual Health Care 2012;24:28-38.

15 Brett J, Staniszewska S, Mockford C, et al. A systematic review of the impact of patient and public involvement on service users, researchers and communities. Patient 2014;7:387-95.

16 National Institute for Health Research. Going the extra mile: improving the nation's health and wellbeing through public involvement in research, 2015. Available: www.nihr.ac.uk/about-us/ documents/ Extra\%20Mile2.pdf [Accessed 3 Dec 2018].

17 National Institute for Health Research. Involve, 2018. Available: https://www.invo.org.uk [Accessed 3 Dec 2018].

18 James Lind Alliance. The James Lind alliance Guidebook (version 8), 2018. Available: http://www.jla.nihr.ac.uk/jla-guidebook/downloads/ Version-8-JLA-Guidebook-for-download-from-website.pdf [Accessed 3 Dec 2018].

19 James Lind alliance. James Lind alliance priority setting partnerships, 2017. Available: www.jla.nihr.ac.uk [Accessed 3 Dec 2018].

20 Boney $\mathrm{O}$, Bell M, Bell N, et al. Identifying research priorities in anaesthesia and perioperative care: final report of the joint National Institute of academic Anaesthesia/James Lind alliance research priority setting partnership. BMJ Open 2015;5:e010006.

21 Fernandez MA, Arnel L, Gould J, et al. Research priorities in fragility fractures of the lower limb and pelvis: a UK priority setting partnership with the James Lind alliance. BMJ Open 2018;8:e023301.

22 National Institute for Health and Care Excellence. Fractures (noncomplex): assessment and management (NG38). London: NICE, 2016.

23 The British Society of Surgery for the Hand. Best practice for management of distal radius fractures (DRFs), 2018.

24 The British Society of Surgery for the Hand. Common conditions of the hand and wrist priority setting partnership, 2017. Available: www.jla.nihr.ac.uk/priority-setting-partnerships/commonconditons-affecting-the-hand-and-wrist/downloads/JLA-FinalSummary.pdf

25 Online surveys (formerly Bristol online surveys), 2018. Available: https://www.onlinesurveys.ac.uk [Accessed 3 Dec 2018].

26 Rangan A, Upadhaya S, Regan S, et al. Research priorities for shoulder surgery: results of the 2015 James Lind Alliance patient and clinician priority setting partnership. BMJ Open 2016;6:e010412.

27 Paskins Z, Jinks C, Mahmood W, et al. Public priorities for osteoporosis and fracture research: results from a general population survey. Arch Osteoporos 2017;12:45. 